\title{
Exercise! Facts related to exercise in individuals with spinal cord lesion
}

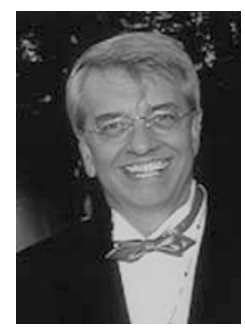

JJ Wyndaele, Editor

Antwerp University Hospital, Antwerp, Belgium

E-mail: spinalcord@uza.be

Dear Spinal Cord reader,

In the review article in this issue, Ploumis et al. evaluate the evidence supporting a role for vasopressor support in the management of acute spinal cord injury and look for updated recommendations regarding the appropriate clinical application of this therapeutic modality. Four questions were studied: what patient groups benefit from vasopressor support, what is the optimal hypertensive drug regimen, what is the optimal duration of the treatment, and what is the optimal arterial blood pressure. Outcome measures used were the incidence of patients needing vasopressors, the increase of arterial blood pressure and neurological improvement. They conclude that there is currently no gold standard on vasopressor support. Based on non-randomized human studies, complete cervical cord injuries require vasopressors more frequently than other spinal cord injuries. More details and the editorial note by Krassioukov can be found with the manuscript.

Research data are provided in the manuscripts of Xiong et al. on motor unit number estimation to evaluate motor function recovery in SCI rats and of Vialle et al. on motor and sensitive axonal regrowth after multiple intercosto-lumbar neurotizations in a sheep model.

Exercise is the topic of several contributions. Lannem et al. found that while perceived fitness is associated with exercise in the whole of the spinal cord injured (SCI)-population, exercisers with a complete lesion reported a much higher perceived exercise mastery and exercisers with incomplete SCI reported a lower perceived exercise mastery than non-exercisers. Anneken et al. found physical exercise to be the main influencing determinant of quality of life, particularly within the physical and psychological dimensions. Leal et al. describe how hydrostatic compression could be the main contributor to improving vital capacity in tetraplegic subjects immersed in water, improvement occurring despite increased plasma volume during immersion.

Ozawa et al. prospectively evaluated 683 patients with cervical myelopathy who underwent decompressive surgery. T1, 2 and Gd-DTPA enhanced MRI were taken before surgery. They found that intramedullary enhancement indicated not the severity of preoperative symptoms, but a sign of a worse prognosis. Benito Penalva et al. examined the effects of transcranial magnetic stimulation (TMS) on the soleus H reflex in SCI patients before and after locomotion training. They conclude that TMS-induced $\mathrm{H}$ reflex-modulation may help in the assessment of changes in the descending control of leg reflexes. The changes on reflex modulation in SCI patients probably occur within the first 3 months after injury. Wirz et al. performed a retrospective analysis of prospectively collected data on outcome in central cord versus Brown-Sequard Syndrome. In contrast to the assumption of a better outcome of subjects with BS, no difference was found between the two incomplete SCI groups. This is of interest with respect to the different potential mechanisms leading to a recovery of functions in these two SCI subgroups.

Lee et al. state, based on their study, that clinicians treating persons with SCI should be aware that fatigue is a common and significant problem that could be exacerbated by the use of medication. van Loo et al. showed substantial unmet care needs in persons with long-term SCI living at home in the Netherlands. Information, psychosocial care and self-efficacy were the areas most in need of improvement.

One interesting case report by Frisbie describes that in an individual with tetraplegia, the partial raising of the head of his bed for a certain period of time recruited increased breathing effort and venous return to the chest, preventing orthostatic hypotension on transfer to an upright position.

Enjoy reading 